\title{
Shp2-mediated molecular signaling in control of embryonic stem cell self-renewal and differentiation
}

\author{
Gen-Sheng Feng ${ }^{1}$ \\ ${ }^{1}$ Programs in Signal Transduction and Stem Cells and Regeneration, Burnham Institute for Medical Research, 10901 N. Torrey Pines \\ Rd, La Jolla, CA 92037, USA
}

A key issue to be addressed in stem cell biology is the molecular signaling mechanism controlling embryonic stem (ES) cell pluripotency. Stem cell properties are dictated by specific transcription factors and epigenetic processes such as DNA methylation and chromatin remodeling. Several cytokines/growth factors have been identified as critical ES cell regulators. However, there is a gap in our knowledge of the intracellular signaling pathways linking extracellular signals to transcriptional regulation in ES cells. This short review discusses the physiological role of Shp2, a cytoplasmic tyrosine phosphatase, in the molecular switch governing ES cell self-renewal versus differentiation. Shp2 promotes ES cell differentiation, mainly through bi-directional modulation of Erk and Stat3 pathways. Deletion of Shp2 in mouse ES cells results in more efficient self-renewal. This observation provides the impetus to develop Shp2 inhibitors for maintenance and amplification of ES cells in culture.

Cell Research (2007) 17:37-41. doi:10.1038/sj.cr.7310140; published online 9 January 2007

Keywords: Shp2, embryonic stem cell pluripotency, embryonic stem cell self-renewal, embryonic stem cell differentiation

\section{Introduction}

Embryonic stem (ES) cells and somatic stem cells have potential therapeutic applications for treating disease. In particular, the recent success of establishing human ES cell lines has ignited great excitement over stem cell research in the biomedical field. However, progress in human ES cell research has been hindered by difficulties in manipulating their self-renewal versus differentiation properties in vitro, largely due to lack of understanding of the molecular and cellular basis of pluripotency. Stem cell properties are ultimately determined by gene expression profiles controlled by specific transcription factors and also by epigenetic processes such as DNA methylation and chromatin remodeling. Transcription factors such as Nanog, Oct4, and Sox 2 play critical roles in stem cells and

Correspondence: Gen-Sheng Feng

Tel: 1-858-713-6265; Fax: 1-858-713-6274

E-mail:gfeng@burnham.org co-occupy a substantial portion of their target genes $[1,2]$. A large set of genes associated with development must be repressed in human ES cells to maintain pluripotency, and their activation leads to ES cell differentiation [3].

Stem cell properties are also tightly regulated by environmental cues, such as cytokines and growth factors. However, there is a gap in our knowledge of the intracellular signaling cascades linking extracellular signals to transcription in stem cells. Accumulating experimental data suggest that mouse and human ES cells employ both shared and distinct signaling pathways to regulate pluripotency, but the complete picture is not yet clear. This review focuses on functional roles of the Shp2 tyrosine phosphatase in signaling events controlling ES cell self-renewal and differentiation.

\section{Shp2, a cytoplasmic tyrosine phosphatase with two SH2 domains}

An important advance in understanding dynamic interactions between protein tyrosine kinases and phosphatases 
(PTKs and PTPs, respectively) in cell signaling is the identification of two mammalian PTPs containing two Src-homology 2 (SH2) domains, Shp1 and Shp2 [4, 5]. Shp1 expression is mainly detected in hematopoietic and epithelial cells, while Shp2, which is homologous to the corkscrew gene product in Drosophila, is ubiquitously expressed. The appearance of SH2 domains in these PTPs suggests roles in signaling events immediately downstream of receptor PTKs. Indeed, both Shp1 and Shp2 bind various receptor PTKs in a variety of cell types. Docking of the PTPs to an activated receptor via $\mathrm{SH} 2$ domains may upregulate the phosphatase activity, an idea substantiated by resolution of the Shp2 crystal structure [6]. This structure predicts that intracellular targeting of the enzyme via the $\mathrm{SH} 2$ domain is directly coupled to stimulation of catalysis, which is a highly efficient regulatory mechanism.

Biological and biochemical data indicate that Shp2 acts as a positive signal transducer between receptor PTKs and the Erk pathway. Ectopic expression of a catalytically inactive Shp $2^{\mathrm{CS}}$ mutant severely suppressed insulin-stimulated Erk activation [7, 8]. Microinjection of a catalytically inactive mutant Shp2 mRNA into Xenopus embryos also blocked FGF-stimulated Erk activation [9]. Targeted deletion of exon 3, encoding 65 amino acids in the SH2-N domain of murine Shp2 (Shp $\left.2^{\Delta 46-110}\right)$, resulted in embryonic lethality in homozygotes with abnormal patterning of mesodermal structures and defects in Erk activation stimulated by growth factor signaling $[10,11]$. However, how Shp2 enhances signaling through the Erk pathway is not fully understood, although several possible mechanisms have been proposed to explain the positive effect of the tyrosine phosphatase in cell signaling [12]. In addition to receptor PTKs, Shp2 is also a signaling component for cytokine receptors that lack intrinsic kinase activity, such as receptors for leukemia inhibitory factor (LIF), interferons, growth hormone, erythropoietin, interleukin-2 (IL-2), IL-6 and IL-11 [13]. Shp2 is also implicated in integrin signaling to control cell adhesion, migration and cytoskeletal architecture [14]. In addition to acting as a positive effector in the Erk pathway, Shp2 also operates in the NF- $\mathrm{BB}$ and Jak-Stat pathways as a negative or positive modulator [15-17].

\section{Shp2 is required for ES cell differentiation to all blood cell lineages}

A critical role of Shp2 in promoting ES cell differentiation into specific cell types was first identified in the hematopoietic cell compartment [18]. As noted, homozygous deletion of exon 3 of the $\operatorname{Shp} 2$ locus leads to embryoniclethality in mice [10]. To dissect Shp2 functions in development of various cell lineages, Qu et al. isolated homozygous Shp2-mutant ES cell lines with the exon 3 deletion, and ex- amined the impact of Shp2 deletion on hematopoiesis [18], using an in vitro ES cell differentiation assay established by Keller's group [19]. In a semi-solid culture system, ES cells grow into cellular spheres termed embryoid bodies (EBs). Within the EB structure, ES cells have the capacity to differentiate into various cell lineages, such as neural, hematopoietic, muscle, and endothelial cells; and therefore, the EB system is a useful tool to study the function of a specific gene product in ES cell differentiation. Directed differentiation of ES cells to specific cell lineages can be facilitated by addition of defined cytokine cocktails or other reagents to the culture medium. As evaluated by the colony-forming unit (CFU) assay, ablation of Shp2 severely suppressed mouse ES cell development to erythroid and myeloid progenitors, suggesting a positive requirement for Shp2 in hematopoiesis [18]. This in vitro observation was supported and substantiated by analysis in vivo, in which Shp2-deficient erythroid or myeloid progenitor cells were barely detectable in the fetal liver or bone marrow of chimeric animals derived from aggregation of mutant ES cells and wild-type embryos [20]. Notably, Shp2-mutant ES cells did contribute to many other tissues of the chimeras, suggesting a more stringent requirement for Shp2 in hematopoiesis than in the development of other cell types in mammals [20]. An essential role for Shp2 in lymphopoiesis was also identified in a Rag2-deficient blastocyst rescue experiment, as development of lymphoid cell lineages in Shp2 $2^{-/} /$Rag- $2^{-/}$chimeric mice was blocked before the pro$\mathrm{T}$ - and pro-B-cell stages [21]. Therefore, Shp2 is positively required for the development of all blood cell lineages. It is important to note that this requirement has been further demonstrated in a rescue experiment, in which wild-type Shp2 was re-introduced into Shp2-mutant ES cells [22]. Reintroduction of Shp2 rescued hematopoiesis as assayed by immunoblotting, ES cell colony differentiation in vitro, and hemoglobinization of primary EBs, respectively. In one-step and two-step EB formation assays, expression of Shp2 restored both primitive and definitive hematopoiesis in Shp2-mutant ES cells.

A requirement for Shp2 in the development of all blood cell lineages indicates that it functions in signaling at a very early step of hematopoiesis, for example in the commitment/differentiation of hematopoietic stem cells (HSCs). This notion was supported by experiments by Chan et al examining expression of early developmental marker genes [22]. As reported previously [23], ES cell differentiation to mesoderm can be assessed by evaluating the marker brachyury. Evaluation of hemangioblast development by assaying for blast-CFC (colony -forming cell) formation and $f k-1$ expression can be used to analyze generation of a multi-potential precursor with the capacity to differentiate into primitive and definitive erythroid and endothelial 
cells [23, 24]. Compared to wild-type ES cells, brachyury transcripts were much lower in day 3 EBs derived from Shp2-deficient ES cells. Consistently, lower frequency of blast-CFC formation and decreased levels of $f k-1$ transcripts were detected in EBs derived from Shp2-mutant ES cells [22]. Therefore, the severe defect in blood cell development from Shp2-mutant ES cells is likely due to an initial block in ES cell differentiation to mesodermal precursors. These findings are consistent with the observed defect in Shp $2^{-/}$embryos, which exhibit abnormal gastrulation and notochord formation [10]. Notably, reconstitution with wild-type Shp2 in Shp2-deficient ES cells rescued brachyury and $f k-1$ expression and also, in part, differentiation to blast-CFC [22].

The unique function of Shp2 in hematopoietic cells has been further underscored by the identification of somatic gain of function mutations in the human PTPN11 gene, which encodes Shp2, in juvenile myelomonocytic leukemia, myelodysplastic syndromes and acute myeloid leukemia [25]. In this respect, PTPN11 is the first identified proto-oncogene that encodes a tyrosine phosphatase [12].

\section{Role of Shp2 in ES cell differentiation to other cell types}

Unlike an essential role for Shp2 in hematopoiesis, the impact of Shp2 deletion on cardiomyocyte development is less severe [26]. In the "hanging drop" culture system, ES cells aggregate, and these ES cell aggregates outgrow and generate spontaneously beating foci following transfer to tissue culture plates. Wild-type ES cells can give rise to autonomously contracting foci at $100 \%$ efficiency $5 \mathrm{~d}$ after re-plating aggregates from the hanging drop culture. However, Shp2-mutant ES cells generated autonomously beating foci two weeks later at an efficiency reduced by approximately 50\%. Consistently, RT-PCR analysis indicates reduced expression of cardiac myosin heavy chain $(M H C)-\alpha$ and $M H C-\beta$ genes in differentiating EBs originating from Shp2-mutant ES cells. Repressed expression of cardiac myosin light chain (MLC2v), myogenin and MyoD was also seen in Shp2-mutant EBs relative to wild-type cells. These results suggest that Shp2 plays a critical role in cardiac muscle cell development and function. More recently, Ke et al. examined neural cell development and found a severe suppression but not a complete block of neuronal/glial cell development from Shp2-deficient ES cells (our unpublished data).

Mouse ES cells are normally maintained in undifferentiated state by addition of LIF to the culture medium. A quick measure of ES cell differentiation capacity is the development of flattened, epithelial/fibroblast-like outgrowth from ES cell colonies upon withdrawal of LIF. Wild-type and
Shp2-mutant ES cells were cultured at low density in LIFcontaining medium and then grown in LIF-deficient medium for 2-3 d. Under this condition, $60-70 \%$ of colonies derived from wild-type ES cells displayed a differentiated morphology, compared to less than 5\% from Shp2-deficient ES cells. Upon reconstitution with wild-type Shp2, the defective differentiation phenotype was rescued, indicating an important role for Shp2 in promoting ES cell differentiation [22].

\section{Elimination of Shp2 leads to improved self-renewal of ES cells in vitro}

Decreased differentiation potential of a mutant ES cell line is not necessarily equivalent to increased self-renewal. Whether deletion of Shp2 alters the self-renewal capacity of ES cells has been determined, in assays for secondary EB formation efficiency $[22,26]$. In the EB structure, ES cells not only differentiate into many committed cell lineages but also undergo limited proliferative self-renewal. Thus, ES cells isolated from EBs at early stages can form secondary EBs when replated. If the self-renewal capacity of a mutant ES cell line is increased, the relative number of ES cells within the EB would be elevated, leading to higher efficiency of secondary EB formation. Notably, although Shp2 deficiency caused a reduced proliferation rate of ES cells, there was a significant increase in the efficiency of secondary EB formation in Shp2-mutant ES cells, as compared to wild-type cells [26]. Re-introduction of wild-type Shp2 into Shp2-deficient ES cells resulted in restoration of secondary EB formation to wild-type levels [22]. Therefore, Shp2 elimination not only suppresses ES cell differentiation, but also leads to increased self-renewal. It is also interesting to note that the absence of functional Shp2 yields a survival advantage to ES cells as a decrease in apoptosis was detected in Shp2-deficient ES cells using annexin V staining [22]. This survival advantage likely contributes in part to the increased frequency of secondary EBs; however, the relative increase in the survival of Shp2mutant ES cells is much less than the increase in secondary EB frequency, and therefore the increased survival alone cannot account for the elevated secondary EB frequency. Shp2 is likely involved in signaling that directly controls ES cell self-renewal and the maintenance of pluripotency.

\section{Shp2 modulates LIF-stimulated Erk and Stat3 sig- nals bi-directionally in mouse ES cells}

Consistent with their reduced differentiation capacity upon LIF withdrawal, Shp2-mutant ES cells are more sensitive than wild-type cells to the differentiation-suppressing effect of LIF. When cultured at gradually reduced concentrations of LIF, Shp2-mutant ES cells maintain a 
morphologically undifferentiated status at a LIF concentration that is 4 -fold less than that required to maintain wild-type ES cells [26]. Consistently, Shp2-deficient ES cells also exhibited enhanced sensitivity to LIF in a "LIF rescue" experiment, in which ES cells are first cultured at low concentrations of LIF for up to $72 \mathrm{~h}$, followed by incubation in normal ES cell medium containing regular amounts of LIF $(1000 \mathrm{U} / \mathrm{ml})$, and are assayed for differentiation in $120 \mathrm{~h}$. After incubation at $60 \mathrm{U} / \mathrm{ml}$ of LIF for $72 \mathrm{~h}$, Shp2-mutant ES cells could fully recover, while wild-type ES cells displayed increased number of differentiated colonies in proportion to the length of incubation time at the low LIF doses.

LIF signals through gp130, a common subunit of the IL-6 family of cytokines, by binding to the heterodimeric LIF receptor-gp 130 complex resulting in activation of the Jak/Stat and Erk pathways. LIF-stimulated activation of Stat 3 is required to maintain ES cells in an undifferentiated and self-renewing state [27-29]. Notably, LIF-stimulated phospho-Stat3 signals are higher in Shp2-deficient ES cells compared to wild-type cells, and expression of wild-type Shp2 in Shp2-deficient ES cells down-regulates LIF-stimulated phospho-Stat 3 levels [22]. This observation suggests a negative role of Shp2 in regulating LIF-stimulated Stat3 activation in ES cells. Consistent with this observation, ES cells engineered to express a G-CSF-gp130 chimeric receptor bearing a mutation in the Shp2 binding tyrosyl residue (Y757) of gp130 required lower levels of gp130 stimulation to maintain pluripotency compared to ES cells expressing a wild-type chimeric receptor [30], although interpretation of this result is complicated by the observation that SOCS-3 (suppressor of cytokine signaling-3) also binds to Y757 of gp130.

In contrast to the increased phospho-Stat 3 signal, LIFstimulated phospho-Erk levels are decreased in Shp2-deficient ES cells, compared to wild-type cells or Shp2-mutant cells reconstituted with wild-type Shp2 expression [22, 26]. Therefore, Shp2 has a reciprocal role in modulating LIF-stimulated Erk and Stat3 pathways in ES cells. It is likely that the balance between Stat 3 activation, which is required for ES cell self-renewal, and Erk activation, which promotes differentiation, determines stem cell fate. Shp2 facilitates ES cell differentiation by down-regulating the Stat3 pathway, while promoting signaling through the Erk pathway (Figure 1). Consistently, other groups have shown that activation of Stat 3 and suppression of Erk signals are critical to maintain mouse ES cells in an undifferentiated status [28-30].

Recent studies suggest that the LIF-Stat 3 pathway is dispensable for maintenance of human ES cell pluripotency [31, 32]. Thus, it is of interest to know if Shp2 plays a similar role in molecular signaling in human ES cells,
A

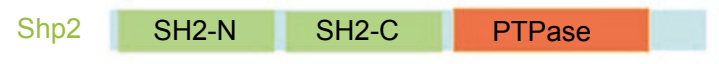

$\mathrm{B}$

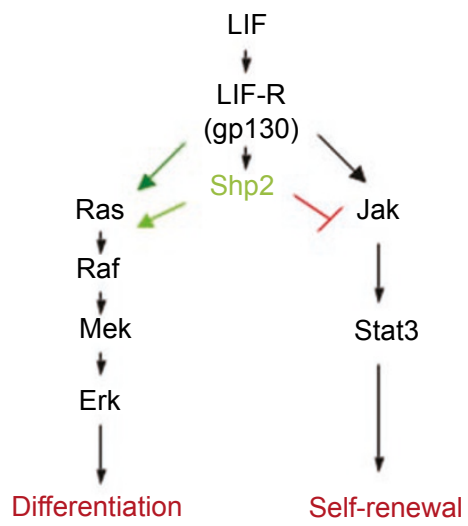

Figure 1 A simplified model on Shp2 functions in ES cells. (A) A schematic structure of Shp2 that contains two SH2 domains at the $\mathrm{N}$-terminus and a single catalytic domain. (B) Shp2 modulates ES cell self-renewal and differentiation via bi-directional regulation of the Erk and Stat3 pathways. However, it is likely that Shp2 has additional roles in other pathways, which are yet to be elucidated.

and our preliminary results suggest that Shp2 does have a similar function in mouse and human ES cells (Wu et al., our unpublished data).

\section{Perspectives}

Phenotypic analysis of Shp2-deficient mouse ES cells indicates a role of Shp2 tyrosine phosphatase in promoting ES cell differentiation into many committed cell lineages. Shp2 appears to modulate LIF-stimulated Erk and Stat3 pathways in opposite directions (Figure 1). However, it is likely that Shp2 modulates additional, yet to be elucidated, intracellular signaling pathways that also contribute to controlling mouse ES cell pluripotency. Using Shp2-deficient mouse ES cells as a model system, we might be able to determine upstream signaling cascades regulating key transcription factors underlying ES cell pluripotency. As elimination of Shp2 in mouse ES cells leads to increased self-renewal, chemicals inhibiting Shp2 may be of potential use in amplification of ES cells in culture. It will also be important to determine the function of Shp2 in the control of human ES cell differentiation and self-renewal, which would help elucidating both common and distinct signaling mechanisms operating in mouse/human ES cells. 


\section{Acknowledgment}

Work on mouse and human ES cells in the author's laboratory has been supported by NIH grants (GM53660, CA78606 and GM075059), and a postdoctoral fellowship from California Institute of Regenerative Medicine (CIRM).

\section{References}

1 Boyer LA, Lee TI, Cole MF, et al. Core transcriptional regulatory circuitry in human embryonic stem cells. Cell 2005; 122:947956.

2 Loh YH, Wu Q, Chew JL, et al. The Oct4 and Nanog transcription network regulates pluripotency in mouse embryonic stem cells. Nat Genet 2006; 38:431-440.

3. Lee TI, Jenner RG, Boyer LA, et al. Control of developmental regulators by Polycomb in human embryonic stem cells. Cell 2006; 125:301-313.

4 Feng GS, Pawson T. Phosphotyrosine phosphatases with SH2 domains: regulators of signal transduction. Trends Genet 1994; 10:54-58.

5 Neel BG, Gu H, Pao L. The 'Shp'ing news: SH2 domain-containing tyrosine phosphatases in cell signaling. Trends Biochem Sci 2003; 28:284-293.

6 Hof P, Pluskey S, Dhe-Paganon S, Eck MJ, Shoelson SE. Crystal structure of the tyrosine phosphatase SHP-2. Cell 1998; 92:441450.

7 Milarski KL, Saltiel AR. Expression of catalytically inactive Syp phosphatase in 3T3 cells blocks stimulation of mitogen-activated protein kinase by insulin. J Biol Chem 1994; 269:2123921243.

8 Noguchi T, Matozaki T, Horita K, Fujioka Y, Kasuga M. Role of SH-PTP2, a protein-tyrosine phosphatase with Src homology 2 domains, in insulin-stimulated Ras activation. Mol Cell Biol. 1994; 14:6674-6682.

9 Tang TL, Freeman RM Jr, O'Reilly AM, Neel BG, Sokol SY. The SH2-containing protein-tyrosine phosphatase SH-PTP2 is required upstream of MAP kinase for early Xenopus development. Cell 1995; 80:473-483.

10 Saxton TM, Henkemeyer M, Gasca S, Shen R, Rossi DJ, Shalaby F, Feng GS, Pawson T. Abnormal mesoderm patterning in mouse embryos mutant for the SH2 tyrosine phosphatase Shp-2. EMBO J 1997; 16:2352-2364.

11 Shi ZQ, Lu W, Feng GS. The Shp-2 tyrosine phosphatase has opposite effects in mediating the activation of extracellular signal-regulated and c-Jun NH2-terminal mitogen-activated protein kinases. J Bio Chem1998; 273:4904-4908.

12 Chan RJ, Feng GS. PTPN11 is the first identified proto-oncogene that encodes a tyrosine phosphatase. Blood 2006. Epub ahead of print.

13 Lai LA, Zhao C, Zhang EE, Feng GS.The Shp-2 tyrosine phosphatase. In: Arino J, Alexander D, eds. Protein phosphatases. Vol. 5. Berlin Heidelberg: Springer-Verlag, 2004: 275-299.

14 Yu DH., Qu CK, Henegariu O, Lu X, Feng GS. Protein tyrosine phosphatase Shp-2 regulates cell spreading, migration and focal adhesion. J Biol Chem 1998; 273:21125-21131.

15 You M, Flick LM, Yu D, Feng GS. Modulation of the Nuclear
Factor kappaB Pathway by Shp-2 Tyrosine Phosphatase in Mediating the Induction of Interleukin (IL)- 6 by IL-1 or Tumor Necrosis Factor. J Exp Med 2001; 193:101-110.

16 You M, Yu DH, Feng GS. Shp-2 tyrosine phosphatase functions as a negative regulator in the interferon-stimulated Jak/STAT pathway. Mol Cell Biol 1999; 19:2416-2424.

17 Ali S, Chen Z, Lebrun J, Vogel W, Kharitonenkov A, Kelly PA, Ullrich A. PTP1D is a positive regulator of the prolactin signal leading to beta-casein promoter activation. Embo J 1996; 15:135-142.

18 Qu CK, Shi ZQ, Shen R, Tsai FY, Orkin SH, Feng GS. A deletion mutation in the SH2-N domain of Shp-2 severely suppresses hematopoietic cell development. Mol Cell Biol 1997; 17:54995507.

19 Keller GM, In vitro differentiation of embryonic stem cells. Curr Opin Cell Biol 1995; 7:862-869.

20 Qu CK, Yu WM, Azzarelli B, Cooper S, Broxmeyer HE, Feng GS. Biased suppression of hematopoiesis and multiple developmental defects in chimeric mice containing Shp-2 mutant cells. Mol Cell Biol 1998; 18:6075-6082.

21 Qu CK, Nguyen S, Chen J, Feng GS. Requirement of Shp-2 tyrosine phosphatase in lymphoid and hematopoietic cell development. Blood 2001; 97:911-914.

22 Chan RJ, Johnson SA, Li Y, Yoder MC, Feng GS. A definitive role of Shp-2 tyrosine phosphatase in mediating embryonic stem cell differentiation and hematopoiesis. Blood 2003; 102:20742080.

23 Choi K, Kennedy M, Kazarov A, Papadimitriou JC, Keller G. A common precursor for hematopoietic and endothelial cells. Development 1998; 125:725-732.

24 Kennedy M, Firpo M, Choi K, Wall C, Robertson S, Kabrun N, Keller G. A common precursor for primitive erythropoiesis and definitive haematopoiesis. Nature 1997; 386:488-493.

25 Tartaglia M, Niemeyer CM, Fragale A, Song X, Buechner J, Jung A, Hahlen K, Hasle H, Licht JD, Gelb BD. Somatic mutations in PTPN11 in juvenile myelomonocytic leukemia, myelodysplastic syndromes and acute myeloid leukemia. Nat Genet 2003; 34:148150.

26 Qu CK, Feng GS. Shp-2 has a positive regulatory role in ES cell differentiation and proliferation. Oncogene 1998; 17:433-439.

27 Raz R, Lee CK, Cannizzaro LA, d'Eustachio P, Levy DE. Essential role of STAT3 for embryonic stem cell pluripotency. Proc Natl Acad Sci USA 1999; 96:2846-2851.

28 Niwa H, Burdon T, Chambers I, Smith A. Self-renewal of pluripotent embryonic stem cells is mediated via activation of STAT3. Genes Dev 1998; 12:2048-2060.

29 Matsuda T, Nakamura T, Nakao K, Arai T, Katsuki M, Heike T, Yokota T. STAT3 activation is sufficient to maintain an undifferentiated state of mouse embryonic stem cells. EMBO J 1999; 18:4261-4269.

30 Burdon T, Chambers I, Nichols J, Smith A. Suppression of SHP-2 and ERK signalling promotes self-renewal of mouse embryonic stem cells. Dev Biol 1999; 210:30-43.

31 Daheron L, Opitz SL, Zaehres H, Lensch WM, Andrews PW, Itskovitz-Eldor J, Daley GQ. 2004. LIF/STAT3 signaling fails to maintain self-renewal of human embryonic stem cells. Stem Cells 22:770-778.

32 Sumi T, Fujimoto Y, NakatsujiN, Suemori H. STAT3 is dispensable for maintenance of self-renewal in nonhuman primate embryonic stem cells. Stem Cells 2004; 22:861-872. 